\title{
Structural health monitoring and damage identification for composite panels using smart sensor
}

\begin{abstract}
Real-time monitoring of structural integrity is an important challenge. This article presents the results of damage detection in real time for two materials: Al 6061-T6 and twill weave carbon fibre-reinforced epoxy composite. The natural frequency as a global dynamic technique was adopted and the structure was evaluated based on the change in the natural frequency. A square thin plate with simply supported edges was investigated under the effect of sinusoidal signal which was generated via mechanical vibration exciter to carry out the natural frequency of the panel. A smart sensor (piezoelectric ceramic lead zirconate titanate) bonded to the surface of the composite panel was used to capture the signals. Experiments demonstrate the effect of change in crack depth and the response of these panels. The results were measured via monitoring technique and evaluated using root mean square deviation index as statistical analysis.
\end{abstract}

Keyword: Structural health monitoring; Damage identification; Twill weave carbon fibre; Composites; Smart sensor; Root mean square deviation 